\title{
Temperature Dependent Anharmonic Properties of Lead Chalcogenides
}

\author{
K.M. RAJU* \\ Department of Physics, Brahmanand Post-Graduate College, Rath, Hamirpur, U.P. 210 431, India
}

(Received December 8, 2009; in final form April 13, 2010)

\begin{abstract}
Calculations were made to investigate the anharmonic properties of lead chalcogenides $\mathrm{PbS}$, $\mathrm{PbSe}$ and $\mathrm{PbTe}$ at elevated temperatures by means of primary physical parameters viz. nearest-neighbour distance and hardness parameter using long- and short-range potentials. Higher-order elastic constants are computed up to their melting temperature for these crystals. The first order pressure derivatives of second- and third-order elastic constants, the second-order pressure derivatives of second-order elastic constants and partial contractions are also evaluated at different temperatures. As the experimental elastic constants are not available at high temperatures, hence our results were only compared with the available values obtained at room temperature. The nature of the elastic behaviour in these compounds was analyzed.
\end{abstract}

PACS numbers: 62.20.D-, 81.40.Jj

\section{Introduction}

Higher order elastic constants and pressure derivatives provide useful information about the inter-ionic potentials and anharmonic properties of crystalline solids. Kailash et al. [1] have recently derived general expressions for the anharmonic properties of $\mathrm{NaCl}$-structure ionic solids following the well-known method of homogeneous deformation. These expressions are easily applicable $[2,3]$ to determine the higher order elastic constants and related properties at temperature near melting point. Nowadays, determination of anharmonic properties at high temperatures [1-6] is of common interest, therefore, we have used these expressions to evaluate the second-, third- and fourth-order elastic constants (SOECs, TOECs and FOECs), the first-order pressure derivatives (FOPDs) of SOECs and TOECs, second-order pressure derivatives (SOPDs) of SOECs and partial contractions of lead chalcogenide crystals crystallising in the NaCl-structure. Since the thermal effects contribute significantly, the temperature dependence of elastic constants is much more sensitive to the short-range interactions. In the present paper we perform an analysis of the higher order elastic constants and pressure derivatives on the basis of the Born-Mayer potentials.

The paper is organized as follows: In Sect. 2, we describe the calculation procedure in brief. Theory is tested for lead chalcogenides in Sect. 3. Results and discussion are presented in Sect. 4 .

\section{Theory}

The elastic energy density for a crystal of a cubic symmetry can be expanded up to quartic terms as shown below [1]:

$$
U_{0}=U_{2}+U_{3}+U_{4}=[1 / 2 !] C_{i j k l} x_{i j} x_{k l}
$$

* e-mail: rajukm@yahoo.com

$$
\begin{aligned}
& +[1 / 3 !] C_{i j k l m n} x_{i j} x_{k l} x_{m n} \\
& +[1 / 4 !] C_{i j k l m n p q} x_{i j} x_{k l} x_{m n} x_{p q}
\end{aligned}
$$

where $C_{i j k l}, C_{i j k l m n}$ and $C_{i j k l m n p q}$ are the SOECs, TOECs and FOECs in tensorial form; $x_{i j}$ are the Lagrangian strain components, $C_{I J}, C_{I J K}$ and $C_{I J K L}$ are the SOECs, TOECs and FOECs in Brügger's definition [7] and Voigt notations [8]. The SOECs, TOECs and FOECs are as given below

$$
\begin{aligned}
& C_{i j k l}=C_{I J}=\left(\partial^{2} U / \partial x_{i j} \partial x_{k l}\right)_{x=0}, \\
& C_{i j k l m n}=C_{I J K}=\left(\partial^{3} U / \partial x_{i j} \partial x_{m n} \partial x_{k l}\right)_{x=0}
\end{aligned}
$$

and

$$
\begin{aligned}
& C_{i j k l m n p q}=C_{I J K L} \\
& \quad=\left(\partial^{4} U / \partial x_{i j} \partial x_{k l} \partial x_{m n} \partial x_{p q}\right)_{x=0} .
\end{aligned}
$$

An elastic constant consists of two parts as follows:

$$
\begin{aligned}
& C_{I J}=C_{I J}^{0}+C_{I J}^{\mathrm{vib}}, \quad C_{I J K}=C_{I J K}^{0}+C_{I J K}^{\mathrm{vib}}, \\
& C_{I J K L}=C_{I J K L}^{0}+C_{I J K L}^{\mathrm{vib}} .
\end{aligned}
$$

The first part is the strain derivative of the internal energy $U_{0}$ and is known as "static" elastic constant and the second part is the strain derivative of the vibrational free energy $U^{\mathrm{vib}}$ and is called "vibrational" elastic constant. The superscript "0" has been introduced to emphasize that the static elastic constants correspond to $0 \mathrm{~K}$. By adding the vibrational elastic constants to the static elastic constants, one may get SOECs and TOECs at any temperature for fcc crystals. The general expressions for these properties have been reported in [1] and therefore are not repeated in this paper.

\section{Evaluation}

Lead chalcogenides are a group of semiconductors consisting of lead and elements of group VI (sulfur, selenium 
and tellurium in particular). Lead chalcogenides share many physical and chemical properties in common [9]. They are isostructural, i.e. have the same crystal structure. They can be prepared and doped by similar methods. Some lead chalcogenide alloys, such as lead telluride and lead selenide, are established as good thermoelectrics. Lead chalcogenides form cubic sodium chloride $(\mathrm{NaCl})$ lattice structure which belongs to $F m 3 m$ space group. Presently, the anharmonic properties for lead chalcogenides are evaluated at 100-1000 K utilizing the concepts of the nearest neighbour distance $(r)$ and hardness parameter $(q)$. These properties are calculated (taking $r=2.970,3.060$ and $3.225 \AA$ for $\mathrm{PbS}, \mathrm{PbSe}$ and $\mathrm{PbTe}$, respectively, and $q=0.345 \AA$ ) at different temperatures using the theory described earlier $[1,7,8]$. The whole evaluation is based on the assumption that the fcc crystal structure of the material does not change when temperature varies up to their melting point. In the calculations we have taken only lattice constant (nearest neighbour distance) as the input parameter and employed only one adjusting [10] hardness parameter $(q)$ [11-13]. This parameter (hardness parameter) is taken as constant for each compound in present study. It is assumed that hardness parameter is independent of temperature and can be taken empirically nearly equal for all the compounds in existing computations. The small variation $[14,15]$ in hardness parameter does not reflect any significant change in elastic constants and related properties of the crystals. Thus it is justified to suppose that the variation of the hardness parameter is insignificant with respect to our calculations. The values of second, third and fourth order elastic constants, first and second order pressure derivatives of SOECs and first order pressure derivatives of TOECs at room temperature for these crystals are given in Tables I-III. The available theoretical and experimental data, compiled from Shanker and Singh [9], are also presented in these Tables for the sake of comparison. Temperature variations of some anharmonic properties for these substances are shown in Figs. 1-8 choosing a few data obtained in this study.

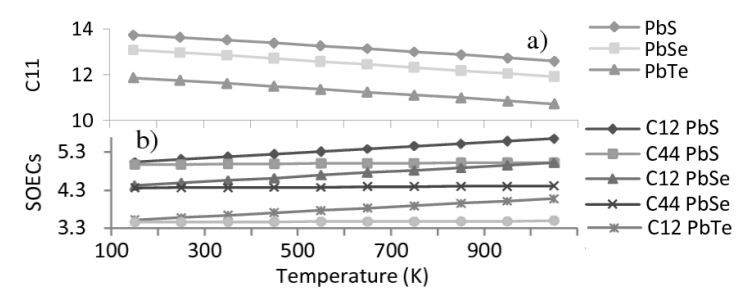

Fig. 1. Temperature variation of SOECs (in $10^{10} \mathrm{~N} / \mathrm{m}^{2}$ ).

TABLE I

The second and third order elastic constants in $10^{10} \mathrm{~N} / \mathrm{m}^{2}$ at room temperature for lead chalcogenides (comparison data from Ref. [9], experimental values are given in bold numerals).

\begin{tabular}{c|c|c|c|c|c|c|c|c|c}
\hline \hline Crystal & $C_{11}$ & $C_{12}$ & $C_{44}$ & $C_{111}$ & $C_{112}$ & $C_{123}$ & $C_{144}$ & $C_{166}$ & $C_{456}$ \\
\hline $\mathrm{PbS}$ & 13.53 & 5.18 & 4.99 & -223.89 & -19.67 & 7.11 & 8.09 & -20.36 & 8.04 \\
& 12.40 & 3.10 & 2.30 & -178.00 & -13.70 & 9.11 & 8.16 & -8.82 & 7.68 \\
$\mathrm{PbSe}$ & 12.85 & 4.56 & 4.37 & -215.83 & -17.13 & 6.19 & 7.18 & -17.81 & 7.14 \\
& 12.40 & 1.93 & 1.59 & -166.00 & -2.13 & 7.46 & 7.05 & -0.13 & 6.85 \\
$\mathrm{PbTe}$ & 11.62 & 3.64 & 3.47 & -201.56 & -3.39 & 4.81 & 5.82 & -4.07 & 5.78 \\
& 10.70 & 0.77 & 1.30 & -143.00 & -2.73 & 5.11 & 5.74 & -5.97 & 6.06 \\
& & & & $\mathbf{- 1 8 5 . 0 0}$ & $\mathbf{- 3 . 5 0}$ & $\mathbf{9 . 7 0}$ & $\mathbf{4 . 4 0}$ & $\mathbf{- 9 . 8 0}$ & $\mathbf{1 . 2 0}$
\end{tabular}

TABLE II

The fourth order elastic constants in $10^{10} \mathrm{~N} / \mathrm{m}^{2}$ at room temperature (comparison data from Ref. [9]).

\begin{tabular}{c|c|c|c|c|c|c|c|c|c|c|c}
\hline \hline Crystal & $C_{1111}$ & $C_{1112}$ & $C_{1122}$ & $C_{1123}$ & $C_{1144}$ & $C_{1155}$ & $C_{1255}$ & $C_{1266}$ & $C_{1456}$ & $C_{4444}$ & $C_{4455}$ \\
\hline $\mathrm{PbS}$ & 257.10 & 34.30 & -33.00 & -45.60 & -1.89 & 9.31 & 1.88 & 11.37 & -1.87 & 11.41 & -1.88 \\
& 2291 & 54.20 & 68.00 & -23.2 & -19.80 & 20.10 & -18.70 & 36.80 & -17.00 & 33.10 & -25.00 \\
$\mathrm{PbSe}$ & 278.30 & -8.70 & -7.60 & -18.60 & -1.68 & 8.06 & -1.67 & 9.99 & -1.66 & 10.02 & -1.66 \\
& 1994 & -13.40 & -1.35 & -18.1 & -16.70 & -26.80 & -16.30 & -13.80 & -15.60 & -15.20 & -18.80 \\
$\mathrm{PbTe}$ & 272.22 & -4.50 & -3.70 & -1.22 & -1.36 & 6.21 & -1.35 & 7.93 & -1.35 & 7.96 & -1.35 \\
& 1681 & -2.99 & 6.51 & -10.6 & -12.90 & -19.60 & -13.70 & -27.20 & -14.80 & 29.70 & -9.48
\end{tabular}

\section{Results and discussions}

An investigation of the higher-order elastic constants and their pressure derivatives provides useful information on the inter-atomic forces, inter-ionic potentials and on anharmonic properties of crystalline solids. This is why, recently, there have been several attempts [1-5, 16-20] to determine the elastic constants of higher order using experimental and theoretical $[1,16,20]$ techniques. In 
TABLE III

The partial contractions (in $10^{12} \mathrm{~N} / \mathrm{m}^{2}$ ), FOPDs and SOPDs (in $10^{-12} \mathrm{~N} / \mathrm{m}^{2}$ ) of the SOECs and FOPDs of the TOECs at $300 \mathrm{~K}$ (comparison data from Ref. [9], experimental values are given in bold numerals).

\begin{tabular}{|c|c|c|c|c|c|c|c|c|c|c|c|c|c|c|c|c|c|}
\hline Crystal & $Y_{11}$ & $Y_{12}$ & $Y_{44}$ & $\frac{\mathrm{d} C_{11}}{\mathrm{~d} P}$ & $\frac{\mathrm{d} C_{12}}{\mathrm{~d} P}$ & $\frac{\mathrm{d} C_{44}}{\mathrm{~d} P}$ & $\frac{\mathrm{d} S}{\mathrm{~d} P}$ & $\frac{\mathrm{d} K}{\mathrm{~d} P}$ & $\frac{\mathrm{d}^{2} C_{11}}{\mathrm{dP}}$ & $\frac{\mathrm{d}^{2} C_{12}}{\mathrm{dP}}$ & $\frac{\mathrm{d}^{2} C_{44}}{\mathrm{~d} P}$ & $\frac{\mathrm{d} C_{111}}{\mathrm{~d} P}$ & $\frac{\mathrm{d} C_{112}}{\mathrm{~d} P}$ & $\frac{\mathrm{d} C_{123}}{\mathrm{~d} P}$ & $\frac{\mathrm{d} C_{144}}{\mathrm{~d} P}$ & $\frac{\mathrm{d} C_{166}}{\mathrm{~d} P}$ & $\frac{\mathrm{d} C_{456}}{\mathrm{~d} P}$ \\
\hline \multirow{2}{*}{$\mathrm{PbS}$} & -4.54 & -37.15 & -6.25 & -9.34 & 1.74 & 0.15 & 3.60 & 4.54 & 13.97 & -3.62 & 0.54 & -47.67 & 48.48 & 57.05 & -1.22 & 17.29 & 2.34 \\
\hline & & & & & & 0.61 & 3.79 & 4.34 & -2.12 & -0.40 & -0.32 & -97.20 & -4.11 & 3.27 & 0.76 & 3.35 & 2.50 \\
\hline $\mathrm{PbSe}$ & & & & & & -1.52 & 4.02 & 3.37 & -1.38 & -0.20 & -0.49 & -87.30 & 1.42 & 2.97 & 0.73 & 4.68 & 2.61 \\
\hline \multirow[t]{2}{*}{$\mathrm{PbTe}$} & 21.65 & -8.35 & -0.44 & -10.36 & 1.64 & 0.08 & 4.18 & 4.77 & 27.92 & -0.18 & 1.77 & -103.5 & 11.91 & 19.62 & -1.20 & 5.17 & 2.22 \\
\hline & & & & & & -0.60 & 4.63 & 4.05 & -3.96 & -0.28 & -0.38 & -98.90 & 0.25 & 2.36 & 0.88 & 3.10 & 3.13 \\
\hline
\end{tabular}

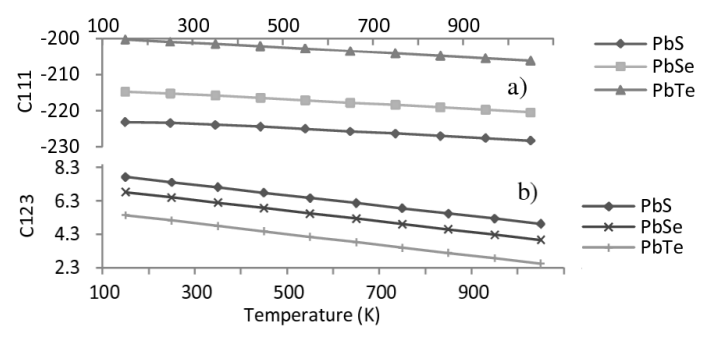

Fig. 2. Temperature variation of TOECs $\left(10^{10} \mathrm{~N} / \mathrm{m}^{2}\right)$.

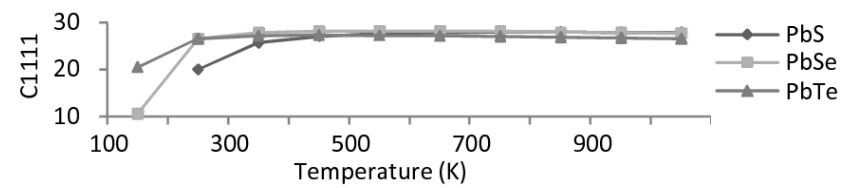

Fig. 3. Temperature variation of a FOEC in $10^{12} \mathrm{~N} / \mathrm{m}^{2}$ for lead compounds.

this paper, the second- and third-order elastic constants at room temperature for lead chalcogenides are shown in Table I together with values calculated by other workers [9]. For the purpose of comparison, experimental values reported in literature are also given in this table (compiled from [9]).

The experimental information on second and third order elastic constants is very scanty for the crystals under study. Only few experimental data are available for TOECs of PbTe. The experimental values of TOECs are not in well agreement with present results but or-

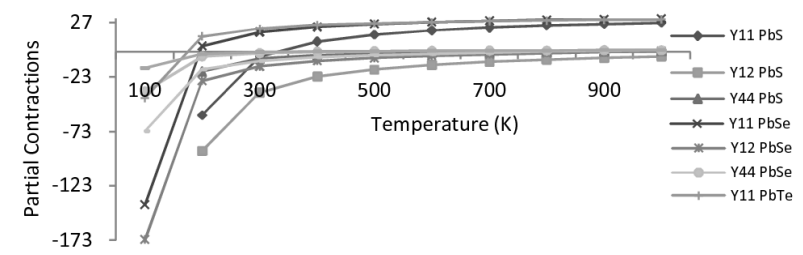

Fig. 4. Temperature variation of partial contractions (in $10^{12} \mathrm{~N} / \mathrm{m}^{2}$ ).

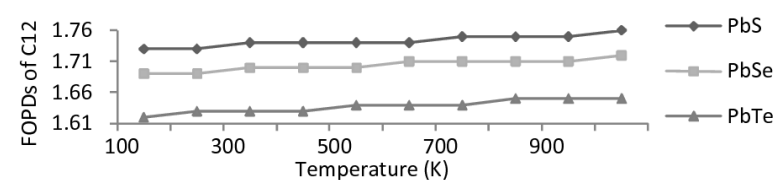

Fig. 5. Temperature variation of FOPD of $C_{12}$.

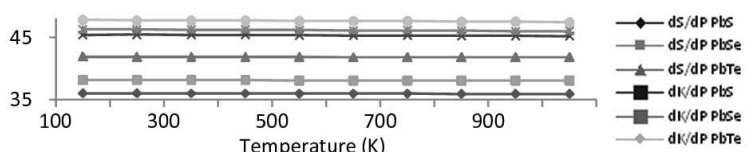

Fig. 6. Temperature dependence $\left(\times 10^{-1}\right)$ of $\mathrm{d} S / \mathrm{d} P$ and $\mathrm{d} K / \mathrm{d} P$.

der of elastic constants is the same. The values of $C_{123}$, $C_{144}$ and $C_{456}$ are positive and are not affected by the inclusion of second-nearest-neighbour interaction to any significant extent suggesting that they are not sensitive to the short-range interaction, whereas other constants $C_{111}, C_{112}$ and $C_{166}$ which comes out negative show a substantial change and are most sensitive to this interaction. Among the calculated TOECs of three materials, $C_{111}$ 's are larger in their absolute values and an order of magnitude larger than the SOECs. Magnitudes of other TOECs are markedly smaller than those of $C_{111}$.

The temperature variations of second and third order elastic constants for chalcogenides of lead are presented in Figs. 1a,b and 2a,b. The data reveal that in going from $100 \mathrm{~K}$ to higher temperatures, the values of second order elastic constants are changed considerably even for

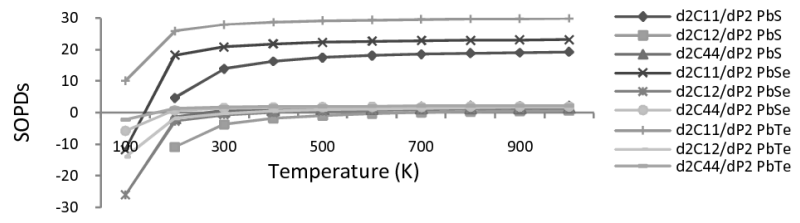

Fig. 7. Variation of SOPDs of SOECS (in $\left.10^{-12} \mathrm{~N} / \mathrm{m}^{2}\right)$. 


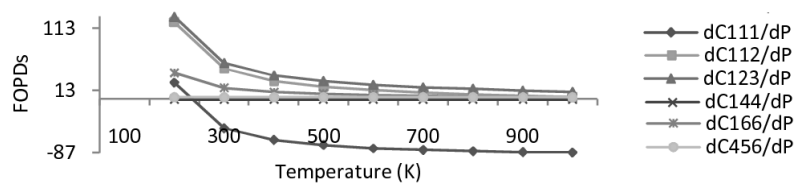

Fig. 8. Temperature variation of FOPD of TOECs for $\mathrm{PbS}$.

highly ionic solids. The values of TOECs increase when temperature is increased. This behaviour is similar to that observed for NaCl-structured solids [1, 3, 16]. Thus our study also proves the previous work. The temperature variation of TOECs could prove useful in studies of various anharmonic properties of ionic solids in general.

Fourth-order elastic constants are required to study many anharmonic properties of crystals and therefore their accurate evaluation is essential. The FOECs at $300 \mathrm{~K}$ for lead chalcogenides are presented in Table II. Some theoretical results [9] are also shown.

Calculated results of fourth order elastic constant $C_{1111}$ at different temperatures are reported in Fig. 3. For NaCl-structure materials we found [1] that the six FOECs, viz. $C_{1111}, C_{1112}, C_{1123}, C_{1122}, C_{1266}$, and $C_{4444}$ are positive and remaining five are negative. This behaviour is also exhibited by other divalent solids like $\mathrm{MgO}, \mathrm{MgS}, \mathrm{CaO}$, SrO etc. under some exemptions. Concerning the FOECs, $C_{1111}$ are the largest and positive in their signs, and an order of magnitude larger than the absolute values of the $C_{111}$ 's. Signs of other fourth order elastic constants are positive or negative and their magnitudes are extremely smaller than the $C_{111}$ 's. Generally, the values of FOECs increase as temperature is increased. There are several theoretical and experimental studies of the FOECs of various crystals [1,9]. Their conclusions reasonably agree with the results of the present calculation. Similar estimation has also been done for magnesium sulphide [16] and lithium halides [21]. There is also a calculation of higher order elastic constants of $\mathrm{MgO}$ [3]. Rigorous calculation of the FOECs of several compounds has been carried out by the present author $[1,3,16,21]$ and the results are not in disagreement with the above estimation. All of these studies conclude that, for various materials, the largest FOECs are positive in sign and an order of magnitude larger than the largest TOEC. After considering these facts, we believe that our values of the calculated anharmonic properties are reliable ones.

The temperature variations of partial contractions are shown in Fig. 4 and comparison has been made in Table III. Due to non-availability of experimental data, the comparison is not completed.

Recently, a number of attempts have been made to calculate the anharmonic properties of ionic crystals [1-6, 16, 19-24]. Only few [5, 6, 16, 19] of them are taken into account in the temperature dependence of these properties. Comparison data on pressure derivatives of SOECs have been reported for all the solids under study and are included in Table III for comparison. Experimental values are available only for few properties and shown in bold numerals in this Table. In contrast our calculated values of FOPDs of SOECs are in close agreement with experiment for all crystals. The general observations made above in the case of lead chalcogenides are true in the other chalcogenides of the similar nature $[5,19]$. The experimental data for FOPDs of SOECs, which are related to the TOECs, have been available for $\mathrm{PbTe}$ and are included in Table III for the sake of comparison. The small deviation of the values at room temperature (Tables I-III) from the experimental data might be due to the fact that these materials are partially covalent in nature. The results can further be improved by taking into account the effect of co-valency in the model potential.

The temperature variations of FOPDs of SOPDs are shown in Figs. 5 and 6 . An important aspect of the present investigation is the calculation of second order pressure derivatives of SOECs at different temperatures. Calculated values of SOPD are available corresponding to $300 \mathrm{~K}$ (Table III). The good agreement between theoretical and experimental values supports the validity of the present work.

Results of second-order pressure derivatives of second-order elastic constants at different temperatures are reported in Fig. 7. Comparison has been made for first order pressure derivatives of third order elastic constants in Table III.

Present results at different temperatures for FOPDs of TOECs for lead chalcogenides are shown in Fig. 8. FOPDs of third order elastic constants are generally decreased as temperature increases. The first order pressure derivatives of SOECs provide a reliable test of a model in the absence of the measured data on TOECs. The values of these pressure derivatives at room temperature predicted by various models are not in good agreement with their experimental data (Table III). A conceivable reason for these deviations may be that their values calculated from the models are subjected to static equilibrium corresponding to $0 \mathrm{~K}$, while the measured data on them are generally available at room temperature.

The data on elastic properties are important because they relate to the various fundamental solid-state phenomena such as inter-atomic potential, equation of state and phonon spectra. Elastic properties are also linked thermodynamically with specific heat, thermal expansion, Debye temperature and Gruneisen parameter. Most importantly, knowledge of elastic constants is essential for many practical applications related to the mechanical properties of solids: load diffraction, thermoelastic stress, internal strain (residual stress) and sound velocity and fracture toughness. We have successfully evaluated temperature dependence of the higher order elastic constants and allied parameters of lead compounds. This paper contains the conclusions on some details of temperature dependent nonlinear properties in lead chalcogenides recently obtained by means of force constant model investigation. Initially the second, third and fourth order 
elastic constants are considered. Then we deal with temperature dependence on pressure derivatives in defining which we use the data on elastic constants.

The calculated and measured values of higher order elastic constants and pressure derivatives for lead chalcogenides are listed in Tables I-III, a look at which reveals that their values obtained by us are much closer to the experimental data than those evaluated by others [9]. However, the general agreements achieved by various workers are such that they do not reveal any clear pattern of superiority for a particular model. The investigation of third- and higher-order elastic constants therefore deserves more thorough treatment. For the phonons of lead chalcogenides, a parameterization within the shell-model $[25,26]$ or recent $a b$ initio calculations [27-29] yields a reasonable agreement with experimental phonon dispersions. Results depend on the parameters for the interaction potentials because the vibrational contribution to the elastic constant contains a sum over all phonons. However, in view of the rigorous derivations and heavy calculations involved in these models, the degree of agreement achieved is not very encouraging since the same can be obtained from present theory which is relatively much simpler and easily adaptable for calculations. The applicability of present model has been tested by several workers by calculating lattice anharmonicity in $\mathrm{LiH}$ and $\mathrm{LiD}$ [30], ultrasonic attenuation in metals [3], chalcogenides [5, 19, 31] and zinc blende structure solids [32]. In view of its simplicity, it appears to be reasonably successful in explaining the basic features of materials. The versatility of this model is further witnessed from its successful applications to explain the semiconducting behaviour [33], nonlinear properties as variation of temperature $[1,3,21,34]$ acoustical parameters of solids $[5,19,32,33,35]$, etc. The theoretical predictions generally show good agreement with measured data for almost all of them. In spite of these versatilities and wide-ranging applicabilities it may be concluded that deformation mechanism used in present model provides much better interpretation of the crystal properties. In the calculations we have taken only lattice parameter and employed only one adjusting hardness parameter. The theory avoids so many assumptions and complexity of the first principle calculation.

Although the experimental values of temperature dependence of elastic constants for lead chalcogenides are not available in the literature, the comparison can be made for the room temperature data in Tables I-III. Thus theoretical calculations seem to be justified. The available literature [36-38] shows that several theories have been used for studying the internal structure and inherent properties of solids. In a general way, rigid shell model and deformation dipole model assume that the electron shells of the ions can suffer deformation or displacements relative to their inner cores. In the deformation dipole model scheme the electron shells are not displaced as a whole but there is a certain redistribution of charges in the deformed electron clouds. The rigid shell-model considers only the displacement of the electron shells with respect to the cores but ignores their deformation although it does take account of the distortion (or mechanical) effect introduced via the short-range overlap forces. This constitutes the main difference between the two models. Neither of them is capable of accounting adequately for the deformability and polarization mechanism simultaneously. These deficiencies impose the necessity of searching a model which can describe both the elastic and dielectric behaviours of solids, satisfactorily. The problem of temperature dependent anharmonic properties in lead chalcogenides cannot be considered as a settled one by now. The results of experimental data analysis show that a general picture of material characterization is rather complicated indeed. This picture cannot be got without researching many-body effects as a whole, with reliable values of input parameters being able to define only after thorough investigation of temperature dependence of elastic and allied properties.

In present work, the order of agreement between calculated and experimental values is reasonably good except in few cases. Expressions obtained in this work are more general than those derived previously $[23,24]$. This treatment gave the possibility to understand the temperature dependences of elastic constants, as well as to secure more exact values of parameters for characterizing the material. The values calculated by the present author seem to be in overall concurrence with the other workers, which is also the same in the case of alkali halides [1]. Full account of many-body interactions between ions and also consideration of the slight non-ionicity of the materials may further improve the results of the calculation. The values reported in this paper could not be compared for elevated temperatures due to the lack of availability of the experimental data on them. Hence, these values are only of academic interest at present and our comments are restricted until the report of measured data on these properties. The present theoretical results on the temperature dependent anharmonic properties of lead compound are probably the first theoretical results of its kind. These parameters may be correlated with the optical and thermoelastic parameters in the broad temperature range. These results, together with other well-known physical properties of these materials, may be expanded as further prospects for the application of lead chalcogenides.

\section{Acknowledgments}

Author is thankful to the reviewers for their valuable comments, which have been used in revising the manuscript. Author is also grateful to Council of Scientific and Industrial Research, New Delhi (India) for financial support.

\section{References}

[1] Kailash,K.M. Raju, S.K. Shrivastava, K.S. Kushwaha, Physica B 390, 270 (2007). 
[2] S.K. Shrivastava, Kailash, K.M. Raju, Ind. J. Phys. 81, 351 (2007).

[3] K.M. Raju, R.K. Srivastava, Kailash, Pramana 69 445 (2007).

[4] P.F. Yuan, Z.J. Ding, Physica B 403, 1996 (2008).

[5] D. Singh, D.K. Pandey, P.K. Yadawa, Cent. Europ. J. Phys. 7, 198 (2009).

[6] S.K. Srivastava, Physica B 387, 396 (2007).

[7] K. Brügger, Phys. Rev. 133, A1611 (1964).

[8] G. Leibfried, H. Hahn, Z. Phys. 150, 497 (1958).

[9] J. Shanker, J.P. Singh, Phys. Status Solidi A 70, 677 (1982).

[10] R.R. Yadav, A.K. Gupta, D. Singh, J. Phys. Studies 9, 227 (2005)

[11] M. Born, J.E. Mayer, Z. Phys. 75, 1 (1932).

[12] M. Born, M.G. Mayer, in: Handbuch der Physik, Vol. 24, Springer Verlag, Berlin 1933.

[13] M.P. Tosi, Solid State Physics, Vol. 16, Academic Press, New York 1965.

[14] Handbook of American Institute of Physics, 2nd ed., Academic Press, New York 1963.

[15] CRC Handbook of Chemistry and Physics, 58th ed., CRC Press, Florida 1977-78.

[16] K.M. Raju, R.K. Srivastava, Kailash, in: Ultrasonics, Macmillan (India) Publishers Pvt. Ltd., New Delhi, 2008, p. 453.

[17] G. Vaitheeswaran, V. Kanchana, S. Heathman, M. Idiri, T. Le Bihan, A. Svane, A. Delin, B. Johansson, Phys. Rev. B 75, 184108 (2007).

[18] S.P. Singh, S. Gupta, S.C. Goyal, Physica B 391, 307 (2007).

[19] R.K. Singh, R.P. Singh, M.P. Singh, S.K. Chaurasia, Turk. J. Phys. 32, 211 (2008).

[20] I. Ohno, K. Harada, C. Yoshitomi, Phys. Chem. Minerals 33, 1 (2006).
[21] Kailash, K.M. Raju, P. Khare, Pure Appl. Ultrason. 28, 181 (2006).

[22] M. Lachhab, D.A. Papaopoulos, M.J. Mehl, J. Phys. Chem. Solids 63, 833 (2002).

[23] V.K. Garg, D.S. Puri, M.P. Verma, Phys. Status Solidi B 82, 325 (1977).

[24] R.K. Varshney, J. Shankar, Phys. Status Solidi B 61, 129 (1985).

[25] W. Cochran, R.A. Cowley, G. Dolling, M.M. Elcombe, Proc. R. Soc. A 293, 433 (1966).

[26] M.M. Elcombe, Proc. R. Soc. A 300, 210 (1967).

[27] K.S. Upadhyaya, M. Yadav, G.K. Upadhyaya, Phys. Status Solidi B 229, 1129 (2002).

[28] A.H. Romero, M. Cardona, R.K. Kremer, R. Lauck, G. Siegle, J. Serrano, X.C. Gonze, Phys. Rev. B 78, 224302 (2008).

[29] O. Kilian, G. Allan, L. Wirtz, Phys. Rev. B 80, 245208 (2009).

[30] R.P. Singh, M.P. Singh, S.K. Chaurasia, R.K. Singh, in Ref. [16], p. 464.

[31] S.K. Kor, D. Singh, A.K. Srivastava, Ind. J. Pure Appl. Phys. 43, 355 (2005).

[32] S.K. Kor, Kailash, J. Phys. Soc. Jpn. 55, 2232 (1986).

[33] D.K. Pandey, D. Singh, R.R. Yadav, Appl. Acoust. 68, 766 (2007).

[34] Kailash, Acta Phys. Pol. A 89, 75 (1996).

[35] S.K. Kor, R.K. Singh, Acta Phys. Pol. A 83, 751 (1993).

[36] R.K. Singh, Phys. Rep. (Rev. Sect. Phys. Lett.) 85, 259 (1982).

[37] S.K. Sinha, CRC Crit. Rev. Solid State Sci. 4, 273 (1973).

[38] W. Cochran, CRC Crit. Rev. Solid State Sci. 2, 1 (1971). 